\title{
Reforma psiquiátrica no Rio Grande do Sul: uma análise histórica, econômica e do impacto da legislação de 1992
}

\author{
Psychiatric reform in Rio Grande do Sul: an analysis of history, economy and the \\ impact of 1992 legislation
}

\author{
Fábio Leite Gastal ${ }^{1}$, Sérgio Olivé Leite ${ }^{2}$, Fabiana Nery Fernandes ${ }^{3}$, Andresa Thier de Borba ${ }^{4}$, \\ Cleyson Makoto Kitamura ${ }^{5}$, Mario Arthur Rockenbach Binz ${ }^{6}$, Milene Tombini do Amaral ${ }^{6}$
}

${ }^{1}$ Psiquiatra. Doutor em Medicina, Universidad de la República Oriental del Uruguay (UROU), Montevidéu, Uruguai. Doutor em Medicina/Psiquiatria,
Universidade Federal de São Paulo (UNIFESP), São Paulo, SP. Professor convidado, Programa de Pós-Graduação em Medicina Tropical, Universidade
Federal de Minas Gerais (UFMG), Belo Horizonte, MG. Superintendente, Organização Nacional de Acreditação (ONA). ${ }^{2}$ Psiquiatra. Professor titular,
Disciplina de Psiquiatria, Universidade Católica de Pelotas (UCPEL), Pelotas, RS. Diretor técnico, Clínica Olivé Leite, Pelotas, RS. ${ }^{3}$ Psiquiatra. ${ }^{4}$
Médica. ${ }^{5}$ Médico. Residente em Oftalmologia, Hospital Banco de Olhos de Porto Alegre, Porto Alegre, RS. ${ }^{6}$ Acadêmicos de Medicina, UCPEL,
Pelotas, RS. Estagiários bolsistas de Iniciação Científica pelo Conselho Nacional de Desenvolvimento Científico e Tecnológico (CNPq).

Este trabalho é vinculado ao Grupo de Avaliação de Serviços, Sistemas de Informações e Políticas de Saúde, CNPq, e foi desenvolvido na Clínica Olivé Leite, em Pelotas, RS.

\section{Resumo}

Os autores apresentam uma análise da reforma psiquiátrica do Rio Grande do Sul, utilizando uma perspectiva histórica e uma abordagem por três vertentes: a psiquiatria institucional; os desenvolvimentos na área extra-hospitalar; e as políticas de saúde implementadas ao longo dos anos. A partir do referencial adotado, foi revisada a experiência brasileira e a do Rio Grande do Sul em particular, tendo como fontes: os bancos de dados MEDLINE e Lilacs e documentos oficiais do Ministério da Saúde e da Secretaria Estadual de Saúde do Rio Grande do Sul.

Hoje, após 14 anos da promulgação da Lei Estadual nº 9716, que dispõe sobre a reforma psiquiátrica no Rio Grande do Sul e determina a substituição progressiva dos hospitais psiquiátricos por uma rede de atenção integral em saúde mental, nota-se que o número de leitos psiquiátricos foi reduzido em $35 \%$, e a proposta de implantação da rede "alternativa" de assistência ao doente mental não foi implementada da forma preconizada, tampouco de maneira igualitária.

Tendo em vista que os atuais desafios, tais como o fenômeno da "porta giratória" e o paciente crônico, refletem a necessidade de extensão dos cuidados primários e secundários, a experiência do Rio Grande do Sul permite pôr em discussão a possibilidade de modificação da estratégia, de tal modo que, ao invés de tentar estruturar um "sistema dentro do sistema”, como hoje está colocado, possa-se tirar melhor proveito da integração da saúde mental aos programas-eixo do SUS, como o Programa de Saúde da Família e o Programa de Agentes Comunitários de Saúde.

Descritores: Psiquiatria, hospital, reforma psiquiátrica, políticas de saúde, avaliação de serviços, economia da saúde.

\begin{abstract}
The authors present an analysis of the psychiatric reform in Rio Grande do Sul, Brazil, using a historical perspective and an approach by three trends: institutional psychiatry; developments in the extra-hospital area; and policies of health implemented along the years. Based on the references adopted, the experience in Brazil and in Rio Grande do Sul was reviewed using the following sources: MEDLINE and Lilacs databases and official documents from the Brazilian Health Ministry and from the Health State Secretary.
\end{abstract}


Nowadays, 14 years after the promulgation of State Law 9716, which refers to the psychiatric reform in Rio Grande do Sul and determines the progressive replacement of psychiatric hospitals by a network of full mental health care, the number of psychiatric beds was reduced in $35 \%$ and the proposal of an "alternative" care network to the mentally ill was not implemented as recommended, neither in an equal form.

Bearing in mind that the present challenges, such as the phenomenon of the "revolving door" and the chronicle patient, show the necessity of extending primary and secondary care. The experience in Rio Grande do Sul brings into debate the possibility of changing the strategy, so that, instead of trying to structure "a system inside the system," as is placed today, more benefits can be drawn from the integration of mental health with the main programs in the Brazilian Unified Health System (SUS), such as Family Health Program (PSF) and Community Health Agents Program (PACS).

Keywords: Psychiatry, hospital, psychiatric reform, health policies, evaluation of services, health economics.

\section{Introdução}

A reforma psiquiátrica insere-se no contexto de transformações ocorridas no setor saúde ao longo dos últimos 50 anos do século XX. Estas estão associadas ao conjunto de mudanças políticas e sociais ocorridas após a Segunda Guerra Mundial, que consolidaram os direitos humanos e a democracia como um valor a ser defendido e preservado. Nesse mesmo conjunto de direitos sociais, a saúde e os direitos dos usuários dos serviços e do paciente psiquiátrico assumem relevância e parte integrante dessa temática.

Paralelamente a essas mudanças políticas e sociais, assiste-se a uma revolução tecnológica que afeta diretamente o setor saúde. Na psiquiatria, essa revolução é representada por enormes progressos na terapêutica, que se iniciam com a penicilina e os antibióticos, que modificam o perfil da demanda dos hospitais psiquiátricos clássicos, ao erradicar de seu universo assistencial as infecções cerebrais e a neurossífilis. Ao mesmo tempo, tem início a moderna psicofarmacologia, que debuta com a clorpromazina nos anos 50 e evolui ao longo dos anos, transformando o perfil epidemiológico e a morbidade das tradicionais doenças mentais.

Essa metamorfose ocorreu, em todos os países, de forma e em ritmos diversos. Tal diversidade pode ser explicada em função das diferentes velocidades de incorporação das novas tecnologias terapêuticas, da capacidade de financiar os sistemas de saúde de cada sociedade em particular e da prioridade dada pelas sociedades aos valores relacionados à democracia e aos direitos humanos e civis.

De qualquer forma, o que se observa hoje é que a reforma psiquiátrica é um processo irreversível e implica em ampla reorganização da atenção à saúde mental, fundamentada na promoção da prevenção, da atenção ambulatorial e emergencial, na psiquiatria hospitalar, tanto em serviços gerais como especializados, bem como em estratégias de reabilitação psicossocial. Essa reformulação deve ter como alicerce a integralidade de ações, sua multidisciplinaridade e universalidade, baseadas no referencial epidemiológico, mas obedecendo aos elementos gerenciais que otimizem a relação custo/ benefício e a qualidade dos serviços organizados, prestados no âmbito do sistema de saúde ${ }^{1}$.

O objetivo deste artigo é analisar a reforma psiquiátrica do Rio Grande do Sul 14 anos após a promulgação da atual legislação, utilizando como eixo uma perspectiva histórica, dados oficiais e institucionais correspondentes a 2002-3 e uma abordagem através de três vertentes: a psiquiatria institucional, os desenvolvimentos na área extra-hospitalar e as políticas de saúde implementadas ao longo do tempo.

\section{História no Brasil}

O setor hospitalar no Brasil, até o final do século XIX, caracterizava-se por uma rede de macro-hospitais públicos, distribuídos principalmente pelas capitais do país e notadamente concentrados na capital federal, Rio de Janeiro. A partir da organização federativa pósrepublicana, inicia-se o processo de descentralização e a criação de serviços organizados em torno dos hospitais de caridade (Santas Casas) e públicos estaduais. Nesse contexto, o hospital psiquiátrico adquire a função de assistência social e abrigo de marginalizados, em detrimento da função médica, terapêutica e reabilitadora ${ }^{2}$.

Nas primeiras décadas do século $\mathrm{XX}$, em Pernambuco, Dr. Ulisses Pernambucano, discípulo do Dr. Juliano Moreira, já discutia os aspectos de humanização da assistência, reabilitação psicossocial, abordagem ambulatorial e pesquisas transculturais, antecipando-se às atuais propostas do movimento de reforma psiquiátrica. Entretanto, na década de 30, com o prenúncio da ditadura Vargas, Dr. Pernambuco é preso e perseguido, em virtude das suas pesquisas sociais, interrompendo, assim, todo um debate em torno dessas questões².

Nos anos 50, observa-se uma mudança significativa no que se refere à questão social e ao custeio da saúde. Surgem as caixas de pensões e os institutos de aposentadorias e pensões, atuando no financiamento da assistência médica de seus segurados. A partir desse fato, observa-se o início da democratização da 
assistência médica no Brasil, do desenvolvimento da seguridade social e do sistema de saúde. A atenção assistencial deixa de ser dicotômica entre pacientes particulares e indigentes e passa a atender, também, aos pacientes segurados, ampliando significativamente sua abrangência social.

Concomitantemente a essas mudanças socioculturais, nota-se importante incremento tecnológico e científico, principalmente a partir da introdução dos antibióticos modernos (penicilina e outros) e dos neurolépticos fenotiazínicos, que deram início à moderna psicofarmacologia, modificando profundamente o perfil epidemiológico dos hospitais psiquiátricos.

Na década de 60, ocorre um aumento da assistência ambulatorial, estimulada pela crescente demanda dos institutos e caixas de pensões, e, em 1964, o golpe militar institui um modelo autoritário e unifica os institutos, criando, assim, a seguridade social nacional unificada e a assistência médica nesse contexto Instituto Nacional de Previdência Social (INPS), Instituto Nacional da Assistência Médica da Previdência Social (INAMPS).

Durante os anos 70, juntamente com o milagre econômico e o crescimento expressivo da população, observa-se o estabelecimento e o desenvolvimento da medicina previdenciária. Porém, no final dessa mesma década e início da seguinte, com a crise econômica generalizada (crise do petróleo, da ditadura e da dívida externa e social) e da seguridade e previdência, iniciase uma fase de graves dificuldades financeiras, administrativas, políticas e ideológicas ${ }^{2}$. O Governo Federal tenta então justificar a crise da Previdência Social, responsabilizando os serviços médicos e prestadores de serviço por todos os problemas do sistema.

Os anos 80 caracterizam-se por uma profunda crise econômica e estrutural do sistema previdenciário nacional, que ocorre concomitantemente à progressiva deterioração das remunerações pagas pela Previdência aos prestadores de serviços. Paralelamente a essas questões, discutia-se a reformulação do sistema de uma maneira geral, e é neste contexto que surge o movimento em prol da denominada "reforma sanitária". Nesse mesmo momento, as discussões internacionais sobre novos modelos assistenciais tiveram repercussão no Brasil, notadamente nos estados de São Paulo, Rio de Janeiro, Minas Gerais e Rio Grande do Sul ${ }^{3}$.

Surgem, então, debates nacionais sobre o papel do hospital psiquiátrico, como na I Conferência Nacional de Saúde Mental, que ocorre em 1987 e tem como temas principais a "reversão da tendência hospitalocêntrica" e o "resgate da cidadania do doente mental". Discutiamse propostas para a reforma psiquiátrica, e, em 1989, é lançado o Projeto de Lei ${ }^{0} 3657$ (Projeto Paulo Delgado - PT/MG), que propunha, em síntese, a implantação de uma rede de assistência extra-hospitalar, a progressiva extinção dos leitos de característica manicomial e a comunicação compulsória das internações involuntárias.

Alguns fatos determinantes para a implementação de uma política de saúde mental, em nível nacional, a partir de 1991, foram a transferência do INAMPS para o Ministério da Saúde e a criação do Sistema Único de Saúde (SUS), através da aprovação da Lei Orgânica da Saúde $n^{\circ} 8080$ e 8142 , de 1990 , que possui como princípios básicos a universalização, a integralidade e a hierarquização das ações, garantindo o acesso à saúde a todos os indivíduos. Antes do SUS, toda a assistência estava vinculada à contribuição previdenciária, excluindo, assim, uma parcela significativa da população.

Ainda no ano de 1990, foi redigida a "Declaração de Caracas”, que propunha a superação do modelo hospitalocêntrico, a humanização dos hospitais psiquiátricos e a ampliação dos direitos dos portadores de transtorno mental. Esse documento opinativo foi fruto da Conferência Regional para a Reestruturação da Assistência Psiquiátrica no Continente, convocada pela Organização Pan-Americana de Saúde (OPAS) e Organização Mundial da Saúde (OMS) e financiada pelo Instituto Mario Negrí, de Milão. As repercussões dessa discussão materializaram-se na forma de Portarias Ministeriais, como as da Secretaria Nacional da Assistência à Saúde - a de nº 189/91, que ampliava e diversificava os procedimentos das tabelas do Sistema de Informações Hospitalares (SIH-SUS) e do Sistema de Informações Ambulatoriais (SIA-SUS) do SUS, e a de ${ }^{\circ}$ 224/92, que estabelecia padrões mínimos para funcionamento dos serviços de assistência à saúde mental no Brasil.

Outros documentos importantes foram as Normas Operacionais Básicas (NOB) 93 e 96 e a Norma Operacional da Assistência à Saúde (NOAS-SUS), de janeiro de 2001, que determina que o cidadão tenha acesso, o mais próximo possível de sua residência, a um conjunto de ações e serviços vinculados a algumas responsabilidades mínimas, dentre as quais o "tratamento dos distúrbios mentais e psicossociais mais freqüentes”.

Em abril de 2001, a Lei ${ }^{\circ} 10.216$ impõe novo impulso e ritmo para a reforma psiquiátrica no Brasil, que dispõe sobre a proteção e os direitos das pessoas portadoras de transtorno mental e redireciona o modelo assistencial em saúde mental, complementando, assim, o Decreto-Lei no 24.559, de julho de 1934.

Com a criação do Programa "De Volta Para Casa", o processo de desinstitucionalização de pacientes longamente internados é acelerado. Tal programa tem 
como objetivo a reintegração social de pessoas acometidas de transtornos mentais, egressas de longas internações, segundo critérios definidos na Lei ${ }^{\circ}$ 10.708, de 31 de julho de 2003, que tem como parte integrante o pagamento do auxílio-reabilitação psicossocial. A regulamentação do programa é a Lei ${ }^{\circ}$ 10.708 junto à Portaria n ${ }^{\circ}$ 2077/GM, de 31 de outubro de 2003.

Ao final do ano de 2001, é realizada, em Brasília, a III Conferência Nacional de Saúde Mental, a qual consolida a reforma psiquiátrica como política de governo, conferindo ao Centro de Atenção Psicossocial (CAPS) o valor estratégico para a mudança do modelo assistencial.

Em 2002, após uma série de normatizações do Ministério da Saúde, o processo de redução de leitos e hospitais psiquiátricos e de desinstitucionalização de pessoas com longa internação ganha grande impulso, sendo que, entre os anos de 2003 e 2005, foram reduzidos 6.227 leitos hospitalares especializados ${ }^{3,4}$.

Além desses marcos fundamentais, houve ainda outras medidas, criadas para normatizar e operacionalizar os novos serviços, como, por exemplo, a Portaria no 824/99 do Ministério da Saúde (MS), que trata da normatização dos atendimentos préhospitalares; a Portaria n ${ }^{\circ} 1077 / 99$ MS, que implanta o programa para aquisição dos medicamentos essenciais para a área de saúde mental; a Portaria ${ }^{\circ}$ 106/00 MS, que cria os serviços terapêuticos; a Portaria $n^{\circ} 44 \mathrm{MS}$, que institui o modelo de assistência do tipo hospital-dia; e a Portaria n ${ }^{\circ}$ 251/02 MS, que estabelece as diretrizes e normas para assistência hospitalar em psiquiatria, reclassifica os hospitais psiquiátricos, define e estrutura a porta de entrada para internações psiquiátricas na rede do SUS e dá outras providências.

\section{Histórico no Rio Grande do Sul}

A psiquiatria gaúcha inicia com a inauguração, na capital, em 1884, do Hospital São Pedro, que, sob forte influência da escola francesa, inaugura, em 1924, o serviço de profilaxia mental do estado. Esse centro constituía a única referência em cuidados aos doentes mentais no estado, até 1931, com a então construção do Sanatório Henrique Roxo (depois Clínica Olivé Leite) em Pelotas.

Apesar da utilização das mais avançadas técnicas terapêuticas da época, como a malarioterapia, o tratamento moral, os barbitúricos, a insulinoterapia, a convulsoterapia, a eletroconvulsoterapia, bem como a laboterapia e a praxiterapia, as internações multiplicavam-se, e a superpopulação e o gigantismo tornaram-se um problema de difícil solução para o Hospital São Pedro.
Na década de 50, tem início o que se pode chamar de a "primeira reforma psiquiátrica", que ocorre com a introdução dos antibióticos e dos psicofármacos na rotina terapêutica. Os medicamentos utilizados desde então permitiram que os doentes mentais saíssem dos hospitais para as comunidades e se integrassem novamente ao convívio de suas famílias.

As mudanças no contexto das internações psiquiátricas ocorreram em todo o mundo, e o Rio Grande do Sul acompanhou essas modificações, sob os auspícios do curso de pós-graduação em Clínica Psiquiátrica da Faculdade de Medicina da Universidade Federal do Rio Grande do Sul (UFRGS). Na década de 60, com um número de 5.500 internos, o Hospital São Pedro inicia seu processo de reforma. Organiza-se, então, a socioterapia, amplia-se a praxiterapia, e cresce a participação multidisciplinar e profissional na equipe de atendimento. $\mathrm{O}$ paciente passa a ser atendido por uma equipe multidisciplinar, sob um enfoque social e familiar e a ótica da psiquiatria dinâmica e do modelo de comunidade terapêutica.

Nesse mesmo período, com base nas experiências internacionais de novos modelos de assistência ao doente mental, alicerçadas principalmente na superação do modelo hospitalocêntrico, surge o movimento que ficou conhecido como a "desinstitucionalização" dos pacientes moradores do Hospital São Pedro, diretamente ligada à evolução histórica da reforma psiquiátrica no estado.

Essa política, continuada por mais de 30 anos e até os dias atuais, influenciou todas as instituições psiquiátricas do estado e, em maior ou menor grau, foi implementada em todo Rio Grande do Sul.

Um exemplo claro dessas mudanças são os dados da Clínica Olivé Leite, localizada em Pelotas. Em sua história, observa-se uma clara evolução na assistência, caracterizada praticamente pela extinção do comportamento asilar, na forma de pacientes cronicamente institucionalizados, que, nos anos 30 , atingia o percentual de quase $18 \%$ dos pacientes hospitalizados, mas que, na década de 90 , não ultrapassa os $0,8 \%$; pela drástica redução do tempo médio de permanência de 256 dias na década de 30 para 130 dias na década de 40, passando a 92 dias nos anos 50, caindo para 57 dias na década de 60, diminuindo para 52 dias nos anos 70 e chegando a 44 dias de permanência em 1985; e, ainda, pela mudança no padrão das altas por óbito, que diminuíram de 3,5\% (década de 30) para $0,01 \%$ na década de $90^{1}$.

Esses dados caracterizam uma profunda reformulação e, na seqüência, evidenciam os fenômenos que serão os grandes desafios para a assistência ao doente mental nos próximos anos: o "novo crônico" e a "porta-giratória", que constituem um grupo de pacientes que é responsável pela metade das internações/ano dos 
serviços, e para o qual é indispensável a formulação de modelos mais adequados de assistência.

No mundo, as discussões sobre a reformulação e melhoria da assistência ao doente mental se multiplicam. Essas idéias tiveram repercussões importantes no estado, como, por exemplo, a inauguração, em 1961, do primeiro hospital-dia do Rio Grande do Sul.

Em 1965, balizado pelo Action for Mental Health, lançado no governo Kennedy, que propunha a psiquiatria comunitária, cria-se o "Plano Crônico", que objetivava a reabilitação dos pacientes crônicos, e o "Plano de Extensão dos Serviços de Assistência Psiquiátrica”, pretendendo desinstitucionalizar o atendimento ao doente mental e promover os conceitos de atendimento preventivos ${ }^{5}$.

Os anos 70 foram particularmente marcantes. Inicia-se o que pode ser chamado de marco inicial da "segunda reforma psiquiátrica do Rio Grande do Sul”: a setorização do atendimento concomitantemente ao plano de interiorização, promovido pela Secretaria de Saúde, que se baseou na contratação de leitos em hospitais privados no interior, na localização de familiares pelo serviço social, em programas de inserção social e na capacitação de médicos e profissionais da área de saúde em todo o estado para receber os egressos com maiores condições técnicas, atendendo, assim, a uma maior demanda, reduzindo o número de novas internações e visando a uma desinstitucionalização progressiva. A partir dessas ações, desospitalizou-se cerca de 65\% dos internos até o início da década seguinte ${ }^{5,6}$.

$\mathrm{Na}$ década de 80 , surge o "movimento antimanicomial”, ou a "terceira reforma psiquiátrica” no estado, que sugere a extinção dos hospitais psiquiátricos, observando-se, contudo, simultaneamente, escassez de recursos e deterioração gradativa da rede comunitária.

Em 1992, através da Portaria ${ }^{\circ} 224$ do Ministério da Saúde, são regulamentados as normas para atendimento e os serviços de assistência em saúde mental.

Até a década de 90, o Rio Grande do Sul não possuía legislação específica sobre a atenção à saúde mental. A partir do movimento antimanicomial, surge a Lei Estadual n ${ }^{\circ}$ 9716, criada em 1992, que dispõe sobre a reforma psiquiátrica e traz as seguintes regulamentações: “fica vedada a construção e ampliação de hospitais psiquiátricos, públicos ou privados e a contratação e financiamento, pelo setor público, de novos leitos nesses hospitais”. A lei determina, ainda, a criação de "variados serviços assistenciais de atenção sanitária e social” e que "a internação psiquiátrica compulsória deverá ser comunicada pelo médico que a procedeu, no prazo de vinte e quatro horas, à autoridade do Ministério Público”. Essa lei passou a ser conhecida como a "lei de reforma psiquiátrica e de proteção aos que padecem de sofrimento psíquico”, e, segundo o seu artigo 15, "no prazo de cinco anos, contados da publicação desta Lei, a reforma psiquiátrica será reavaliada quanto aos seus rumos e ritmo de implantação”.

\section{Situação atual no Rio Grande do Sul}

Com a regulamentação da Lei $n^{\circ}$ 9716/92, o modelo e a assistência psiquiátrica deixam de ser predominantemente hospitalares e passam à atenção psiquiátrica extra-hospitalar, permitindo apenas internações quando indispensáveis e pelo menor prazo de tempo possível.

Segundo o artigo 2 da Lei n 9716/92, “a reforma psiquiátrica consistirá na gradativa substituição do sistema hospitalocêntrico de cuidados às pessoas que padecem de sofrimento psíquico por uma rede integrada de variados serviços assistenciais e atenção sanitária e social, tais como ambulatórios, emergências psiquiátricas em hospitais gerais, leitos ou unidades de internação psiquiátricas em hospitais gerais, hospitaisdia, hospitais-noites, centro de convivência, centros comunitários, centro de atenção psicossocial, centros residenciais de cuidados intensivos, lares abrigados, pensões públicas comunitárias, oficinas de atividade construtivas e similares".

Em 2002, através do Guia de Saúde Mental, a Secretaria Estadual do Rio Grande do Sul propõe uma rede de atenção à saúde mental, composta pela atenção básica em saúde mental, que tem a unidade de saúde (posto de saúde) como referência e desenvolve ações com ênfase na promoção da prevenção, do tratamento e da reabilitação realizados por médico clínico, psicólogo, enfermeiro, assistente social, auxiliar de enfermagem e pelo Programa de Agentes Comunitários de Saúde (PACS) e Programa de Saúde da Família (PSF). Da mesma forma, é realizada a atenção especializada em saúde mental, que serve de contra-referência para apoio, assessoria ou supervisão aos serviços de atenção básica. Para tanto, reúne serviços como ambulatórios gerais com equipe de saúde mental, ambulatórios especializados em saúde mental, CAPS, hospital-dia, urgência e emergência em saúde mental, leitos e unidades psiquiátricas em hospital geral e, por fim, os serviços residenciais terapêuticos temporários ${ }^{7}$. Aspectos mais específicos de cada tipo de serviço estão descritos na Tabela 1. 
Tabela 1 - Tipos de serviços em saúde mental propostos pelo Guia de Saúde Mental do Rio Grande do Sul

\begin{tabular}{|c|c|}
\hline Serviços & Características \\
\hline $\begin{array}{l}\text { Atenção básica } \\
\text { em saúde mental }\end{array}$ & $\begin{array}{l}\text { Ações em saúde mental desenvolvidas pelasunidades de saúde, PACS e PSF, realizadas por médico } \\
\text { clínico, psicólogo, enfermeiro, assistente social e auxiliar de enfermagem, de forma contínua e } \\
\text { personalizada, com ênfase na promoção, prevenção, tratamento e reabilitação, servindo assim, de } \\
\text { porta de entrada ao sistema de saúde para o usuário de saúde mental. }\end{array}$ \\
\hline \multirow{8}{*}{$\begin{array}{l}\text { Atenção } \\
\text { especializada } \\
\text { em saúde mental }\end{array}$} & $\begin{array}{l}\text { A atenção é preferencialmente de contra-referência para apoio, assessoria e supervisão à atenção } \\
\text { básica, que se subdivide em: }\end{array}$ \\
\hline & $\begin{array}{l}\text { Ambulatório com equipe de saúde mental: unidades de saúde que oferecem atenção integral à saúde } \\
\text { incluindo o programa de saúde mental realizado por equipe especializada composta por médico } \\
\text { psiquiatra, psicólogo e assistente social. }\end{array}$ \\
\hline & $\begin{array}{l}\text { Ambulatório especializado em saúde mental: unidades de saúde com equipe multiprofissional } \\
\text { especializada em saúde mental, composta por médico psiquiatra, médico clínico, psicólogo, } \\
\text { enfermeiro, assistente social, terapeuta ocupacional, fonoaudiólogo, neurologista e pessoal auxiliar. }\end{array}$ \\
\hline & $\begin{array}{l}\text { CAPS: serviço ambulatorial de atenção diária com atendimento prioritário aos pacientes com } \\
\text { transtornos mentais severos e persistentes, realizado por equipe multiprofissional especalizada, com } \\
\text { área física independente de qualquer estruturahospitalar. Esse serviço ainda é subclassificado em } \\
\text { CAPS I, II, III, Álcool e Drogas e Criança e Adolescentee, de acordo com a sua complexidade, inclui } \\
\text { ainda leitos (até cinco) para observação e desintoxicação, com permanência máxima de } 7 \text { dias. }\end{array}$ \\
\hline & $\begin{array}{l}\text { Hospital-dia: recurso intermediário entre a internação e o ambulatório, desenvolvendo atenção de } \\
\text { cuidados intensivos, por equipe multiprofissional, visando à substituição da internação integral e } \\
\text { situado em área específica, independente da estrutura hospitalar. As atividades dohospital-dia e do } \\
\text { CAPS se assemelham, exceto que ohospital-dia está vinculado ao hospital geral e o CAPS é uma } \\
\text { unidade ambulatorial independente. }\end{array}$ \\
\hline & $\begin{array}{l}\text { Urgência e emergência em saúde mental: atendimentos de urgência e emergência realizadospor } \\
\text { equipe multiprofissional em serviços de pronto-socorro e/ou pronto-atendimento, situados em } \\
\text { hospitais gerais que atendam } 24 \text { horas por dia com possibilidade de permanência em leitos de } \\
\text { observação por até } 72 \text { horas. }\end{array}$ \\
\hline & $\begin{array}{l}\text { Leitos ou unidades psiquiátricas em hospitais gerais: retaguarda hospitalar para casos em que a } \\
\text { internação é necessária, depois de esgotadas as possibilidades extra-hospitalares. }\end{array}$ \\
\hline & $\begin{array}{l}\text { Serviços residenciais terapêuticos: serviços de assistência de caráter provisório, que visam à } \\
\text { reabilitação psicossocial e à reintegração à família dos usuários oriundos de internações psiquiátricas } \\
\text { longas ou repetidas e/ou em situações de vulnerabilidade social. }\end{array}$ \\
\hline
\end{tabular}

CAPS = Centro de Atenção Psicossocial; PACS = Programa de Agentes Comunitários de Saúde; PSF = Programa de Saúde da Família.

Dados retirados do Guia de Saúde Mental 2002, da Coordenadoria de Saúde Mental do Rio Grande do Sul.

Nos primeiros anos do século XXI, começam a ser percebidos os efeitos deletérios da redução dos leitos hospitalares e da insuficiente estruturação dos modelos alternativos. Surgem movimentos da sociedade civil a exigir a revisão da legislação de 92 e destacando a necessidade de reabertura de vagas e leitos especializados $^{8}$.

Na $3^{\text {a }}$ Coordenadoria Regional de Saúde (CRS) e, mais especificamente, no município de Pelotas, foi desenvolvido pela prefeitura, ao longo dos anos 80 e 90, um esforço de integração da equipe de saúde mental na rede de atenção primária e nos postos de saúde. Segundo a Coordenadoria de Saúde Mental da $3^{\mathrm{a}}$ CRS, em 2002, Pelotas dispunha de 54 postos de atenção primária, dos quais 25 eram atendidos pelo "Serviço Volante de Saúde Mental”, um programa de visitas mensais, realizadas por equipe especializada, aos postos de saúde, para atendimentos especializados e supervisionamento da atenção primária em saúde mental.

Os benefícios da incorporação da assistência psiquiátrica à atenção básica de saúde podem ser observados na comparação entre Pelotas e Rio Grande, ambas as cidades situadas no Rio Grande do Sul, em estudo que demonstrou forte impacto da estruturação de serviços de cuidados em saúde mental na rede de 
atenção primária, sobre o perfil das internações psiquiátricas, principalmente na redução das reinternações dos pacientes esquizofrênicos. Nele, observou-se uma inversão nas prevalências das admissões dos pacientes homens esquizofrênicos e alcoolistas entre as duas cidades. Rio Grande, que dispõe de uma rede limitada, composta apenas por um hospital psiquiátrico e um pequeno ambulatório especializado, apresentou uma prevalência hospitalar de 36 pacientes esquizofrênicos e 25 alcoolistas por 1.000 habitantes; e Pelotas, que possuía, na época da realização do estudo, uma rede assistencial ampliada, equipada com nove equipes - compostas, cada uma delas, por um psiquiatra, um assistente social e um psicólogo, atuando em 12 postos de atenção primária e dois ambulatórios especializados, um grupo trabalhando em um centro integrado de saúde mental para estudantes e adolescentes e, por fim, um outro grupo responsável pelo cuidado dos sem-moradia nos abrigos noturnos -, apresentou 16 pacientes esquizofrênicos e 34 alcoolistas por 1.000 habitantes 9

Esses dados revelam um outro aspecto a ser abordado, que é o problema relacionado ao alcoolismo em homens. Esses pacientes apresentam o transtorno de maior prevalência (15\%) dentro da psiquiatria e podem vir a ocupar todos os leitos anteriormente usados por pacientes esquizofrênicos. Esse fenômeno revela uma carência de políticas de saúde pública visando ao problema do alcoolismo, mesmo onde já existe uma rede assistencial comunitária de saúde mental ${ }^{9-11}$.

Segundo as informações da secretaria do estado, os serviços assistenciais de atendimento a doentes mentais, em meados de 2002, eram distribuídos de acordo com a Tabela 2.

Tabela 2 - Distribuição dos serviços em saúde mental no Rio Grande do Sul

\begin{tabular}{|c|c|c|c|c|c|c|c|}
\hline$\overline{C R S}$ & $\mathbf{A B}$ & $\mathbf{A E}$ & Infantil & CAPS & $\mathbf{A B}+\mathbf{E S M}$ & PS & Moradia \\
\hline $1^{\mathrm{a}} \mathrm{CRS}$ & 12 & 18 & 6 & 4 & 4 & 2 & 1 \\
\hline $2^{\mathrm{a}} \mathrm{CRS}$ & 29 & 11 & 0 & 0 & 0 & 0 & 0 \\
\hline $3^{\mathrm{a}} \mathrm{CRS}$ & 1 & 8 & 0 & 7 & 0 & 0 & 0 \\
\hline $4^{\mathrm{a}} \mathrm{CRS}$ & 22 & 3 & 0 & 0 & 0 & 0 & 0 \\
\hline $5^{\mathrm{a}} \mathrm{CRS}$ & 45 & 6 & 0 & 2 & 0 & 0 & 0 \\
\hline $6^{\mathrm{a}} \mathrm{CRS}$ & 24 & 40 & 1 & 1 & 1 & 0 & 0 \\
\hline $7^{\mathrm{a}} \mathrm{CRS}$ & 3 & 3 & 0 & 0 & 0 & 0 & 0 \\
\hline $8^{\mathrm{a}} \mathrm{CRS}$ & 7 & 2 & 0 & 0 & 0 & 0 & 0 \\
\hline $9^{\mathrm{a}} \mathrm{CRS}$ & 4 & 7 & 0 & 2 & 0 & 0 & 0 \\
\hline $10^{\mathrm{a}} \mathrm{CRS}$ & 1 & 8 & 0 & 0 & 0 & 0 & 0 \\
\hline $11^{\mathrm{a}} \mathrm{CRS}$ & 18 & 1 & 0 & 0 & 0 & 0 & 0 \\
\hline $12^{\mathrm{a}} \mathrm{CRS}$ & 18 & 3 & 0 & 0 & 0 & 0 & 0 \\
\hline $13^{\mathrm{a}} \mathrm{CRS}$ & 7 & 4 & 0 & 1 & 0 & 0 & 0 \\
\hline $14^{\mathrm{a}} \mathrm{CRS}$ & 20 & 2 & 0 & 0 & 0 & 0 & 0 \\
\hline $15^{\mathrm{a}} \mathrm{CRS}$ & 13 & 13 & 0 & 0 & 0 & 0 & 0 \\
\hline $16^{\mathrm{a}} \mathrm{CRS}$ & 40 & 1 & 0 & 0 & 0 & 0 & 0 \\
\hline $17^{\mathrm{a}} \mathrm{CRS}$ & 15 & 4 & 0 & 1 & 0 & 0 & 0 \\
\hline $18^{\mathrm{a}} \mathrm{CRS}$ & 15 & 8 & 0 & 0 & 0 & 0 & 0 \\
\hline $19^{\mathrm{a}} \mathrm{CRS}$ & 2 & 25 & 0 & 0 & 0 & 0 & 0 \\
\hline Total & 296 & 167 & 7 & 18 & 5 & 2 & 1 \\
\hline
\end{tabular}

$\mathrm{AB}$ = atenção básica em saúde mental; $\mathrm{AB}+\mathrm{ESM}$ = atenção básica com equipe de saúde mental; AE = atenção especializada em saúde mental; CAPS = Centro de Atenção Psicossocial; CRS = Coordenadorias Regionais de Saúde; Moradia = serviço de residência terapêutica temporária; PS = pronto-atendimento.

Através dos dados apresentados pela Coordenadoria de Saúde Mental do Rio Grande do Sul, pode-se observar que existe uma concentração de atendimentos extra-hospitalares na $1^{\text {a }}$ CRS, que corresponde à Grande Porto Alegre, concentrando, em geral, os serviços em um determinado município. Assim, aproximadamente 15\% dos municípios do estado não possuem qualquer tipo de atendimento em saúde mental, contrariando, dessa maneira, a Normal Operacional da Assistência à Saúde (NOAS-SUS), 
que preconiza que o acesso seja o mais próximo da residência do paciente. Tal situação obriga a maioria dos pacientes a se deslocar, muitas vezes, por longas distâncias para receber atendimento psiquiátrico.

Um ponto importante a ser abordado é a função atribuída aos CAPS e aos hospitais-dia, que, segundo o Guia da Saúde Mental da Secretaria da Saúde do Rio Grande do Sul, "representam um recurso intermediário entre a internação e o ambulatório, desenvolvendo atenção de cuidados intensivos, por equipe multiprofissional, visando à substituição da internação integral”; porém, o que se observa é o desmonte da rede hospitalar, sem a implantação de um modelo substitutivo adequado, que supra a demanda da população, visto que os hospitais-dia, se existissem, nem estariam relacionados pela Secretaria da Saúde do Rio Grande do Sul, e os CAPS ainda são em número insuficiente e mal distribuídos entre as diversas regiões do estado.

Outra observação que desperta a atenção é o fato de os serviços de urgência e emergência psiquiátricos apenas serem cadastrados como tal quando estão localizados nas dependências de um hospital geral, excluindo, assim, por exemplo, dois serviços de pronto-atendimento psiquiátrico vinculados a hospitais psiquiátricos situados em Pelotas, nos quais cerca de $40 \%$ dos atendimentos são prestados a pessoas provenientes de outros municípios.

Em relação à rede hospitalar, o que se nota é que o número de leitos psiquiátricos disponíveis no estado sofreu um decréscimo importante nos últimos anos, equivalente a uma variação negativa de $35 \%^{12-}$ ${ }^{15}$. Esse fenômeno foi uma das repercussões da Lei $n^{\circ}$ 9716, que “determina a substituição progressiva dos leitos nos hospitais psiquiátricos por rede de atenção integral em saúde mental”.

A distribuição dos leitos destinados à psiquiatria, em meados de 2002, pode ser observada na Tabela 3 , em que se assinalam as diversas referências a serem observadas, tais como a população total de cada CRS do Rio Grande do Sul, os leitos distribuídos tanto em hospitais gerais como nos especializados, o número de leitos preconizados pelo Ministério da Saúde (cerca de 0,5 leitos/1.000 habitantes) e pela OMS (cerca de 1 leito/1.000 habitantes) e, ainda, a diferença entre o número total de leitos disponíveis e o número ideal segundo o Ministério da Saúde.

Tabela 3 - Distribuição de leitos psiquiátricos no Rio Grande do Sul

\begin{tabular}{|c|c|c|c|c|c|c|c|}
\hline CRS & População & Leitos HE & Leitos HG & Total & MS & OMS & Saldo \\
\hline $1^{\text {a }} \mathrm{CRS}$ & 3.381 .924 & 566 & 95 & 661 & 1.691 & 3.382 & -1.030 \\
\hline $2^{\mathrm{a}} \mathrm{CRS}$ & 710.975 & 0 & 30 & 30 & 355 & 711 & -325 \\
\hline $3^{\mathrm{a}} \mathrm{CRS}$ & 842.245 & 469 & 26 & 495 & 421 & 842 & 74 \\
\hline $4^{\mathrm{a}} \mathrm{CRS}$ & 532.009 & 0 & 35 & 35 & 266 & 532 & -231 \\
\hline $5^{\mathrm{a}} \mathrm{CRS}$ & 954.055 & 380 & 18 & 398 & 477 & 954 & -79 \\
\hline $6^{\mathrm{a}} \mathrm{CRS}$ & 564.395 & 56 & 25 & 81 & 282 & 564 & -201 \\
\hline $7^{\mathrm{a}} \mathrm{CRS}$ & 216.597 & 0 & 17 & 17 & 108 & 216 & -91 \\
\hline $8^{\mathrm{a}} \mathrm{CRS}$ & 193.331 & 0 & 6 & 6 & 96 & 193 & -90 \\
\hline $9^{\mathrm{a}} \mathrm{CRS}$ & 135.998 & 0 & 2 & 2 & 68 & 136 & -66 \\
\hline $10^{\mathrm{a}} \mathrm{CRS}$ & 558.455 & 0 & 41 & 41 & 279 & 558 & -238 \\
\hline $11^{\mathrm{a}} \mathrm{CRS}$ & 217.755 & 0 & 5 & 5 & 109 & 218 & -104 \\
\hline $12^{\mathrm{a}} \mathrm{CRS}$ & 243.090 & 0 & 20 & 20 & 121 & 243 & -101 \\
\hline $13^{\mathrm{a}} \mathrm{CRS}$ & 308.323 & 0 & 14 & 14 & 154 & 308 & -140 \\
\hline $14^{\mathrm{a}} \mathrm{CRS}$ & 228.830 & 0 & 22 & 22 & 114 & 229 & -92 \\
\hline $15^{\mathrm{a}} \mathrm{CRS}$ & 162.679 & 0 & 8 & 8 & 81 & 162 & -73 \\
\hline $16^{\mathrm{a}} \mathrm{CRS}$ & 338.244 & 0 & 15 & 15 & 169 & 338 & -154 \\
\hline $17^{\mathrm{a}} \mathrm{CRS}$ & 221.303 & 0 & 12 & 12 & 110 & 221 & -98 \\
\hline $18^{\mathrm{a}} \mathrm{CRS}$ & 287.748 & 0 & 6 & 6 & 144 & 288 & -138 \\
\hline $19^{\mathrm{a}} \mathrm{CRS}$ & 212.065 & 0 & 6 & 6 & 106 & 212 & -100 \\
\hline Total & 10.310 .021 & 1.471 & 403 & 1.874 & 5.155 & 10.310 & -3.277 \\
\hline
\end{tabular}

CRS = Coordenadorias Regionais de Saúde; HE = hospital especializado em psiquiatria; HG = hospital geral infantil: unidades de atenção à criança e ao adolescente; MS = Ministério da Saúde; OMS = Organização Mundial da Saúde. 
Assim como nos serviços extra-hospitalares, também há uma distribuição desigual de leitos entre os municípios. É alto o déficit de leitos na $1^{\text {a }} \mathrm{CRS}$ - cerca de 1.030 -, enquanto que, em todas as outras CRS, o valor resultante também é negativo. A $3^{\mathrm{a}} \mathrm{CRS}$ é a única que possui leitos suficientes, de acordo com o Ministério da Saúde; em conseqüência, recebe pacientes de todas as outras coordenadorias. Contudo, em 2003, foram retirados da rede 199 leitos, com o fechamento de mais um hospital especializado no estado.

Analisando o que foi exposto, verifica-se que o Rio Grande do Sul possui, de fato, um reduzido número de leitos. De acordo com o Ministério da Saúde, o Rio Grande do Sul deveria ter, em 2001, 5.155 leitos e, de acordo com a Organização Mundial da Saúde, 10.310 leitos. Entretanto, existiam apenas 1.874, ou seja, 0,18 leitos para cada 1.000 habitantes. Desde 1993, observa-se uma diminuição no número de leitos em hospitais especializados, passando de 2.262 em 1993 para 1.471 em 2002. Apesar de o número de leitos em hospitais gerais ter aumentado de 366 leitos em 1993 para 403 leitos em 2002, essa elevação ainda é pequena e não supre as necessidades de cada coordenadoria ${ }^{7,16}$.

Da mesma forma, se abordado pela ótica do financiamento em 1999, o Rio Grande do Sul mobilizou cerca de 3\% do total nacional dos gastos do SUS com a psiquiatria, o que equivale, aproximadamente, ao mesmo que Goiás $(3,4 \%)$, que possui metade da sua população, e menos da metade que Pernambuco (6,7\%), com população semelhante ${ }^{17}$.

O custo da Autorização de Internação Hospitalar (AIH) psiquiátrica, em 1999, foi de R\$ 510,07, constituindo uma das menores do país, ficando abaixo da média nacional de $\mathrm{R} \$ 538,65^{17}$.

A psiquiatria gaúcha vem diminuindo a sua participação percentual sobre o gasto total nacional das internações em geral. Em 1999, essa porcentagem foi de $3,5 \%$ sobre os gastos hospitalares (gasto aproximadamente igual ao do Distrito Federal - 3,1\% -, que possui 1/5 de sua população), enquanto a média brasileira foi de 9,4\% e apresentou uma variação negativa de $20 \%$ entre 1995 e $1999^{17}$.

O gasto per capita em psiquiatria por habitantes/ ano, em 1999, foi igual a R \$ 1,36 (o Rio de Janeiro foi o estado com maior gasto, igual a R \$ 5,59), estando o Rio Grande do Sul entre um dos 10 menores do Brasil. Em 2001, apresentou um gasto per capita de R $\$ 1,33$, ficando bem abaixo da média nacional, que foi de R\$ 2,76. No período de 1997 a 2001, o Rio Grande do Sul apresentou uma variação negativa de $11,9 \%$ no gasto per capita em psiquiatria ${ }^{11,12,14,16,17}$.

Além disso, observa-se, em relação aos gastos com hospital-dia, estagnação semelhante quanto aos recursos empregados. Em 1996, foram de R \$175.000,00 e, em
1999, permaneciam nesse mesmo patamar, porém observa-se uma variação negativa quanto à porcentagem destinada a esse serviço pelo SUS, que em 1996 correspondia a 3,2\% do total nacional e em 1999 era de apenas $1,7 \%$. Dados mais atualizados, divulgados recentemente, demonstram que, em 2000, esse montante foi reduzido para $\mathrm{R} \$ 155.234,00$ (gastando mais apenas que Pernambuco - R\$ 122.000,00 - e Mato Grosso do Sul-R\$96.000,00), representando, entre 1995 e 2000, um decréscimo de $11,4 \%$, enquanto o Brasil, nesse mesmo período, apresentou um acréscimo de 206,77\% nos gastos com hospital-dia ${ }^{17,18}$.

O percentual de gastos com hospital-dia no Brasil sobre o total das AIH foi de $0,2 \%$ em 1999, enquanto, no Rio Grande do Sul, permaneceu, desde 1997 até 1999, correspondendo a 0\% do total de AIH nacionais, ficando atrás de estados como Piauí (0,9\%), que possui menos de 1/3 da população do Rio Grande do Sul, e do Distrito Federal (1,0\%), correspondente a $1 / 5$ da população do estado ${ }^{17}$.

Os gastos per capita em hospital-dia, em 1999, foram iguais a $\mathrm{R} \$ 0,02$, enquanto a média brasileira foi de $R \$ 0,06$. Observa-se um marcante déficit em comparação às outras regiões do país, como o Piauí, que apresentou, em 1999, um gasto per capita de R\$ 0,26, 13 vezes maior que o Rio Grande do Sul, ou o Distrito Federal, com R\$ 0,31 ${ }^{18}$.

No ano 2000, a freqüência de AIH com hospitaldia foi igual a 291, estando à frente apenas de Pernambuco (191) e Mato Grosso do Sul (154), e, entre 1995 e 2000, o Rio Grande do Sul apresentou uma variação positiva de $58,9 \%$, enquanto o Brasil apresentou uma variação positiva de $202,56 \%{ }^{18}$.

No Rio Grande do Sul, o número de AIH psiquiátricas, em 2000, foi de 27.977, um volume menor que o apresentado por Goiás (29.218 AIH), que possui a metade da população do estado ${ }^{18}$.

O número de internações psiquiátricas, no ano 2000, foi de 18.440 e apresentou uma variação de + 8,5\% entre 1995 e 2000. Porém, quando se faz o cálculo de internações psiquiátricas por 1.000 habitantes, observa-se que o Rio Grande do Sul encontra-se entre os estados que menos interna, apresentando, em 1995, uma taxa de aproximadamente 1,8 internações-ano/ 1.000 habitantes e permanecendo nesse índice em 2000. Quando comparado aos outros estados, notam-se marcantes diferenças, como, por exemplo, o Rio de Janeiro, que, em 2000, apresentou 4,1 internações-ano/ 1.000 habitantes. Os gastos anuais com internações psiquiátricas no Rio Grande do Sul, em 2000, foram de R\$ 12.797.202,00, apresentando uma variação positiva, entre 1995 e 2000, de $0,7 \%$ (neste mesmo período, a população do estado cresceu $4 \%$ ). $\mathrm{O}$ aumento do gasto nacional foi de $7,2 \%$ (a população brasileira, neste período, cresceu $5 \%)^{17,18}$. 


\section{Comentários finais}

Podemos concluir, após análise desses dados, que o estado do Rio Grande do Sul gasta muito pouco com a psiquiatria. Além disso, possui serviços de baixo custo, quando comparado ao restante do país, apresentando baixas taxas de internação psiquiátrica e diminuição progressiva dos gastos.

Hoje, após mais de 10 anos de promulgação da Lei $n^{\circ} 9716$, o que se nota é a escassez de leitos (segundo os critérios da OMS e do próprio Ministério da Saúde), tanto em hospitais especializados como em hospitais gerais. A proposta de implantação da rede extrahospitalar de assistência ao doente mental não foi concretizada de maneira igualitária, e a reavaliação que deveria ter ocorrido no prazo de 5 anos após a publicação da Lei da Reforma Psiquiátrica não foi realizada.

Existem, porém, aspectos positivos, como a rotina de comunicação das internações compulsórias ao Ministério Público, que oferece maior segurança para o trabalho médico e para o paciente, ficando estes mais protegidos pelos serviços de assistência pública.

Apesar das falhas na concretização da Lei da Reforma Psiquiátrica, o Rio Grande do Sul, atualmente, gasta menos e é mais eficiente que a maioria dos estados da federação. Isso se deve, principalmente, a algumas peculiaridades que existiram no estado e que proporcionaram a progressiva desospitalização, a descentralização e a multidisciplinaridade no atendimento ao doente mental. Nos dias de hoje, evidenciam-se resultados de 30 anos de intervenção voltada para a comunidade e, ainda, para uma ampliação natural do acesso aos serviços e distribuição de medicamentos básicos.

Durante a história, observou-se que não existem modelos que possam ser panacéias ou soluções de âmbito universal, pois, para uma nova proposta assistencial, é necessário considerar fatores socioculturais, econômicos e peculiaridades de cada região e de cada país. Tendo em vista tais princípios e os propostos pela Declaração de Caracas - "a reestruturação da atenção psiquiátrica ligada à Atenção Primária de Saúde e nos marcos dos Sistemas Locais de Saúde permite a promoção de modelos alternativos centrados na comunidade e nas redes sociais" - é que surge a proposta da incorporação de ações de saúde mental na atenção básica a uma rede diversificada de serviços composta pelo PACS e pelo PSF, acompanhada por um plano eficaz de distribuição de medicamentos.

Esse breve histórico evidencia que a reforma psiquiátrica já se realizou no Rio Grande do Sul em termos do componente da desospitalização ou do "desmonte" da rede hospitalar. A tônica da reforma, no caso do Rio Grande do Sul, foi a redução do parque hospitalar e dos investimentos, porém, ao longo da década, não houve a configuração adequada dos espaços e recursos "alternativos" preconizados.

Esse atraso na implantação, reconhecidamente negativo, pode ensejar uma vantagem, pois hoje, com a implantação do SUS e seus programas em larga escala no país, poderia permitir uma reorientação do modelo e a integração da saúde mental às práticas e programas gerais do sistema (PSF e PACS).

A experiência e o exemplo da zona sul do Rio Grande do Sul permitem colocar em discussão a possibilidade de modificação da estratégia, de tal maneira que, ao invés de tentar estruturar um "sistema dentro do sistema", como está hoje colocado através de CAPS, por exemplo, possase tirar melhor proveito e melhor relação custo/benefício da integração da saúde mental aos programas-eixo do SUS, tais como o PSF e o PACS.

Hoje, os desafios e os problemas são similares e estão colocados pela necessidade de extensão de cuidados primários e secundários, fazendo frente aos novos desafios, tais como os fenômenos da "porta giratória" e do paciente crônico em suas novas manifestações, enfrentados, também, por países como Itália, França, Estados Unidos, Espanha e Inglaterra.

Pessoas com distúrbios mentais, apesar do que proclamam os antipsiquiatras, existem e não devem ser ignorados. Os hospitais psiquiátricos são alternativas terapêuticas eficazes e necessárias em situações de crise e devem ser remodelados, modernizados e não extintos. A idéia de que a presença de um serviço hospitalar especializado impediria o avanço para um modelo adequado de assistência é um ledo engano, esclarecido pela experiência internacional e do Rio Grande do Sul dos anos 70 e 80 em particular, na qual a psiquiatria e a atenção em saúde mental caminham sinergicamente com as instituições psiquiátricas.

\section{Agradecimentos}

Ao Fundo de Incentivo ao Desenvolvimento do Ensino e da Pesquisa em Saúde do Ministério da Saúde do Brasil (FIDEPS) pelo financiamento e à professora Nize Terezinha Martins Antunes pela revisão ortográfica dos originais.

\section{Referências}

1. Gastal FL, Leite SO, Gameiro MA, Gastal CL. A reforma psiquiátrica e as organizações não governamentais. Temas. 1993;46:203-16.

2. Gastal FL. Controle estatístico de processo: um modelo para avaliação da qualidade de serviços de internação psiquiátrica [tese]. São Paulo: Universidade Federal de São Paulo; 1995.

3. Alves DN. A reestruturação da atenção em saúde mental no Brasil. Texto apresentado em simpósio no V Congresso da Associação Mundial de Reabilitação Psicossocial; 1996 Abril; Roterdã: Holanda; 1996. 
4. Brasil, Ministério da Saúde, Secretaria de Atenção à Saúde, Departamento de Ações Programáticas Estratégicas, Coordenação Geral de Saúde Mental. Reforma psiquiátrica e política de saúde mental no Brasil. Brasília: Documento apresentado à Conferência Regional de Reforma dos Serviços de Saúde Mental : 15 anos depois de Caracas. OPAS. Brasília: Ministério da Saúde; 2005.

5. Schreen HI, Vernetti CL, Cheffe EA, Fensterseifer G, Faria RN, Silva WEG. A reforma psiquiátrica no Rio Grande do Sul: história da assistência em saúde mental e situação atual. R Psiquiatr. 1997;19(1):8-15.

6. Piccinini WJ. Breve história da psiquiatria do Rio Grande do Sul à luz das suas publicações. R Psiquiatr. 1999;21(2):99-103.

7. Rio Grande do Sul, Secretaria de Saúde. Guia de saúde mental. Porto Alegre: Secretaria da Saúde; 2002.

8. Sonenreich C. Comentários em relação à entrevista do Prof. Valentim Gentil Filho. Temas. 2005;35(68/69):126-34.

9. Andreoli SB, Gastal FL, Lapolli C, Ballester DAP, Daltoe PR, Gameiro M, et al. Deshospitalización psiquiátrica: una perspectiva epidemiológica. Psiquiatria y Salud Integral. 2001;1(2):31.

10. Andreoli SB, Almeida Filho N, Coutinho EF, Mari JJ. Identificação de casos psiquiátricos em estudos epidemiológicos multifásicos: métodos, problemas e aplicabilidade. Rev Saude Publica. 2000;34(5):475-83.
11. Andreoli SB, Gastal FL, Lapolli C, Ballester DAP, Daltoe PR, Gameiro M, et al. O impacto dos cuidados em saúde mental na rede de atenção primária sobre a hospitalização do paciente psiquiátrico: uma visão epidemiológica. Rev Bras Epidemiol. 2002;1:433.

12. Brasil, Ministério da Saúde. Relatório final da Oficina de Inclusão de Ações de Saúde Mental no Programa Saúde da Família. Brasília: Ministério da Saúde; 2002.

13. Brasil, Ministério da Saúde. Números da assistência à saúde no Brasil. Brasília: Ministério da Saúde; 2002.

14. Brasil, Ministério da Saúde. Políticas de saúde. 2002. Disponível em: http://www.saude.gov.br.

15. Brasil, Ministério da Saúde. Rede assistencial. 2002. Disponível em: http://datasus.gov.br.

16. Rio Grande do Sul, Secretaria da Saúde e do Meio Ambiente. A saúde pública e o meio ambiente no Rio Grande do Sul. Porto Alegre: SSMA; 1993.

17. Brasil, Ministério da Saúde, Secretaria de Assistência à Saúde. Assistência hospitalar no SUS - 1995/1999. Brasília: Ministério da Saúde; 2000.

18. Brasil, Ministério da Saúde, Secretaria de Assistência à Saúde. Assistência à saúde no SUS: média e alta complexidade - 1995/2000. Brasília: Ministério da Saúde; 2001. 